University of Nebraska - Lincoln

DigitalCommons@University of Nebraska - Lincoln

July 1976

\title{
Systematic Evaluation of Cellular Radiosensitivity Parameters
}

\author{
R. A. Roth \\ University of Nebraska-Lincoln \\ S. C. Sharma \\ University of Nebraska-Lincoln, sharma@uta.edu \\ Robert Katz \\ University of Nebraska-Lincoln, rkatz2@unl.edu
}

Follow this and additional works at: https://digitalcommons.unl.edu/physicskatz

Part of the Physics Commons

Roth, R. A.; Sharma, S. C.; and Katz, Robert, "Systematic Evaluation of Cellular Radiosensitivity Parameters" (1976). Robert Katz Publications. 127.

https://digitalcommons.unl.edu/physicskatz/127

This Article is brought to you for free and open access by the Research Papers in Physics and Astronomy at DigitalCommons@University of Nebraska - Lincoln. It has been accepted for inclusion in Robert Katz Publications by an authorized administrator of DigitalCommons@University of Nebraska - Lincoln. 


\title{
Systematic Evaluation of Cellular Radiosensitivity Parameters
}

\author{
R. A. Roth, S. C. Sharma, ${ }^{*}$ and Robert Katz \\ University of Nebraska-Lincoln, Lincoln, NE 68508, U.S.A. \\ * Present address: University of Texas System, Cancer Center, \\ Texas Medical Center, Houston, Texas 77025, U.S.A. \\ Corresponding author - R. Katz
}

\begin{abstract}
Cellular radiosensitivity parameters of the track structure theory of Katz and coworkers are evaluated from a sum of squares minimizing computer program for nonlinear models. Based on these observations, suggestions are presented for efficient experiment design for the determination of these parameters from tracksegment bombardments of high LET radiations.
\end{abstract}

\section{Introduction}

In the extension of the $\delta$-ray theory of track structure from nuclear emulsions and other 1-hit detectors to biological cells, a model was achieved in which a set of four experimentally evaluated radiosensitivity parameters is used to calculate cellular survival as a function of absorbed dose in any radiation environment whose particle-energy distribution (quality) is known (Katz, Ackerson, Homayoonfar, and Sharma 1971; Katz and Sharma 1973).

Two of the radiosensitivity parameters for a particular cell line in a particular ambience are obtained from the measured survival curve after $\gamma$-irradiation, and are the extrapolation number of the $\gamma$-ray survival curve, $m$ (nominally interpreted as being associated with the multiplicity of subcellular sensitive sites), and the extrapolated $D-37$ dose, $E_{0}$ (interpreted as related to the radiosensitivity of the sensitive sites when exposed to secondary and higher generation electrons). The other two radiosensitivity parameters, $\sigma_{0}$ and $\kappa$, are found from survival measurements after track-segment irradiations with high LET radiations. The first of these is the plateau (or saturation) value of the inactivation cross-section and nominally represents the cross-sectional area of the cell nucleus, while the second is obtained from the array of available high LET bombardments, for the maximal value of the RBE is expected to occur in the neighborhood of $z^{2} / \kappa \beta^{2}=2$, while the plateau value of the cross-section is achieved when this product is about 4 , when the cell culture is bombarded with beams of particles of effective charge number $z$ at relative speed $\beta$. The quantity $\mathrm{K}$ is understood to reflect the linear dimension of the sensitive sites in the cell, just as $z^{2} / \beta^{2}$ reflects the stopping power of the incident ions. Then $z^{2} / \kappa \beta^{2}$ reflects the energy deposited in a sensitive site.

As in the case of nuclear emulsions, we distinguish between the grain-count regime, where inactivations occur along a particle's path like beads randomly distributed on a 
string, and the track-width regime, where the inactivations are distributed like a "hairy rope" centered on the ion's path. The transition from the grain-count to the track-width regime takes place in the neighborhood $z^{2} / \kappa \beta^{2}=4$; at lower values we are in the graincount regime, at higher values in the track-width regime.

In order to accommodate the capacity of cells to accumulate sub-lethal damage, two modes of inactivation are identified, called "ion-kill" and "gamma- kill." Cells inactivated by the passage of a single heavy ion are said to be inactivated in the ion-kill mode, with inactivation cross-section $\sigma$, whose value is less than $\sigma_{0}$ in the grain-count regime, and greater than $\sigma_{0}$ in the track-width regime. The fraction of "track-segments" inactivating cells in the ion-kill mode in the grain-count regime is said to be equal to o/ $\sigma_{0}$, which is taken to be equal to $P$, the probability for inactivation in the ion-kill mode, and also taken to equal the fraction of the dose deposited in the ion-kill mode. Cells not inactivated in the ion-kill mode are damaged by the $\delta$-rays from the passing particle, and can then be inactivated by $\delta$-rays from other passing ions in the gamma-kill mode, so called because it resembles the mechanism for inactivation by secondary electrons from $\gamma$-ray photons.

In the grain-count regime, the surviving fraction of a cellular population, whose radiosensitivity parameters are $m, E_{0}, \sigma_{0}$, and $\kappa$, after track-segment irradiation with a dose $D$ of a fluence $F$ of particles of atomic number $Z$, effective charge number $z$, relative speed $\beta$, and stopping power $L(\mathrm{LET} \infty)$ is found from the expressions

$$
\begin{aligned}
& N / N_{0}=\Pi_{i} \times \Pi_{\mathrm{\gamma}} \\
& \text { ion-kill mode } \\
& \Pi_{i}=\exp (--\sigma F) \\
& \text { gamma-kill mode } \quad \Pi_{Y}=1-\left\{1-\exp \left[(1-P) D / E_{0}\right]\right\}^{m} \\
& \text { and } \quad P=\sigma / \sigma_{0}=\left[1-\exp \left(-z^{2} / \kappa \beta^{2}\right)\right]^{m}
\end{aligned}
$$

In the track-width regime, where $P>0.98$, we take

$$
\Pi_{\mathrm{Y}}=1
$$

and find $\sigma$ from the "track width" (Figure 2 of Katz and Sharma 1973). As in emulsion, the track width increases linearly with $z / \beta$, and the inactivation cross-section with $z^{2} / \beta^{2}$, up to a limit set by the maximal radial penetration of $\delta$-rays.

In all cases

$$
D=F L
$$

and

$$
z=Z\left[1-\exp \left(-125 \beta Z^{-2 / 3}\right)\right]
$$

From survival data obtained with bombardments at a range of LET values we have extracted cellular radiosensitivity parameters by visual methods. The values of the "best fitting parameters" are shown in Table 1. These are adjusted from an initial set of parameters found ( $m$ and $E_{0}$ ) from the survival curve after X-rays (where $\left.P=0\right),\left(\sigma_{0}\right)$ from the survival curve after a high LET bombardment (where $P \simeq 1$, say $Z=9$ at $10 \mathrm{MeV} / \mathrm{amu}$ ), 


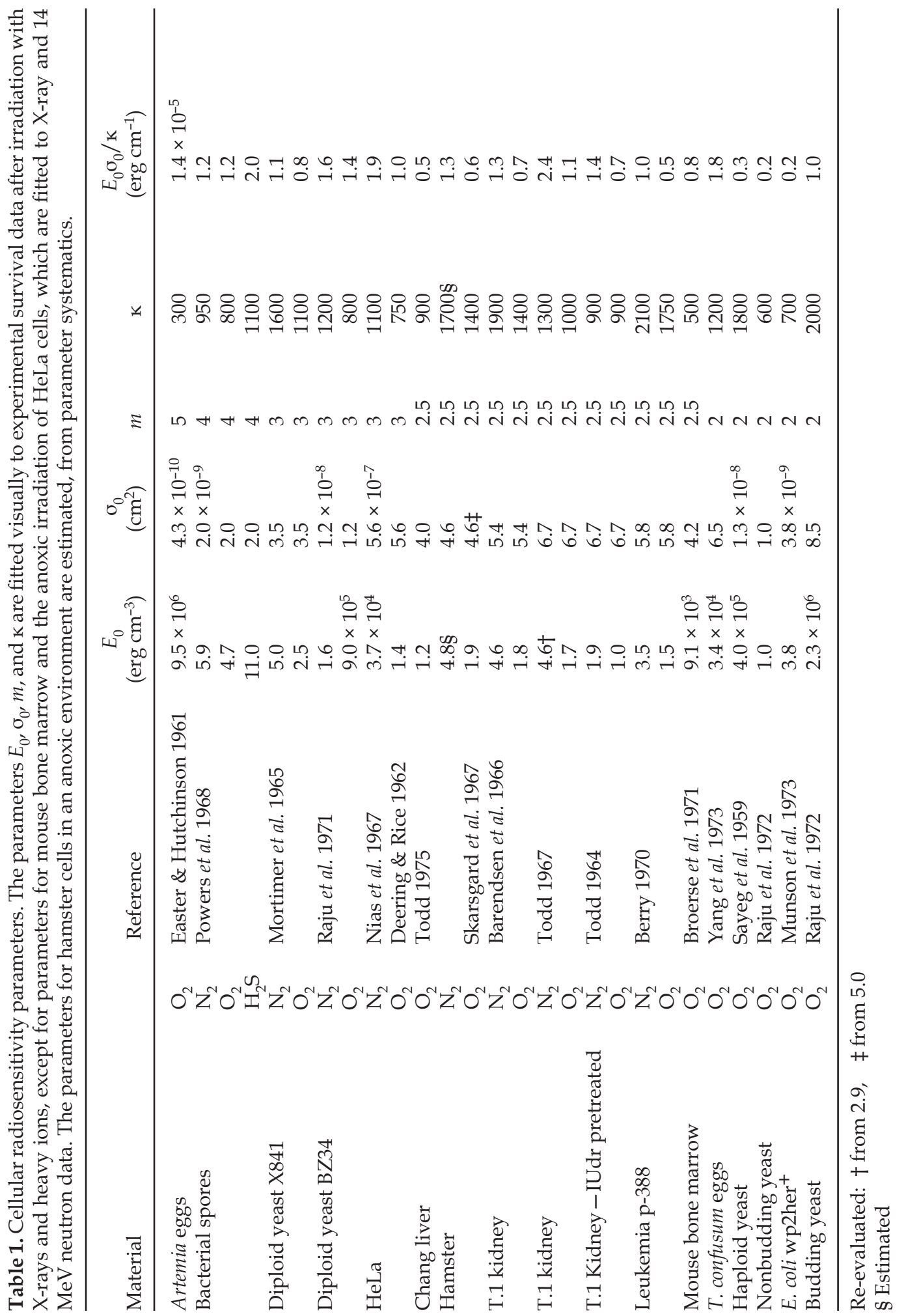


and $(\mathrm{K})$ from the survival curve indicating a maximal $\mathrm{RBE}_{\text {extrapolated }}$ (where $P \simeq 0.5$ ). We are reminded that in the grain-count regime

$$
\mathrm{RBE}_{\text {extrapolated }}=\left(\sigma_{0} E_{0} / L\right) P+(1-P)
$$

The facility with which visually fitted parameters may be assigned depends on the quality of the data. The most serious problems arise when there are no X-ray data from which to make independent assignments of $m$ and $E_{0}$, and no sufficiently high LET data, for then the estimates of $\sigma_{0}, \mathrm{~K}$, and $m$ interact and cannot be considered independently, as seen in equation (4).

To assign parameters on a quantitative basis, with an evaluation of confidence limits, we have applied a sum of squares minimizing computer program to the system of equations and the data. The program (J. P. Ochsner and J. M. Eakman, private communication) makes use of a modified Marquardt method for fitting parameters to a nonlinear model, and here incorporates both grain-count and track-width regimes.

\section{Curve fitting}

Fitting parameters to nonlinear models by least squares is based on the assumption of regions of local linearity in the nonlinear relationship. It is assumed that there is a functional relationship between a dependent variable, one or more independent variables, and the parameters. The independent variables $X_{i}$ are assumed to be accurately measurable, and the dependent variable $Y$ is expressed as (Marquardt 1963)

$$
Y=f\left(X_{1}, X_{2}, \ldots, X_{m} ; B_{1}, B_{2}, \ldots, B_{k}\right)
$$

where the $B_{i}$ are the parameters to be estimated. We consider the experimental data to be a sample taken from an infinitely large population of possible data. Characteristics of the sample are used to estimate those of the population, so that the parameters determined from the sample $\left(b_{i}\right)$ are differentiated from the true values which characterize the population $\left(B_{i}\right)$. Corresponding to each experimental point $\left(Y_{i}, X_{1 i}, X_{2 i}, \ldots, X_{m i}\right)$, a theoretical value

$$
\hat{Y}\left(X_{1 i}, X_{2 i}, \ldots, X_{m i} ; b_{1}, b_{2}, \ldots, b_{k}\right)
$$

is calculated, and the sum of squares of their differences is found as

$$
\Phi=\sum_{i=1}^{n}\left(Y_{i}-\hat{Y}_{i}\right)^{2}
$$

where $n$ is the number of experimental points.

For the linear case, $\Phi$ is minimized by taking the partial derivatives of equation (10) with respect to each parameter, and setting them equal to zero.

In the nonlinear case the minimum, or least squares, is obtained by iteration.

The method of Marquardt is a modified steepest descent method, in which the neighborhood of the minimum is reached by a method of steepest descent, while the best convergence in this region is found by application of a Taylor series expansion to linearize 
the model in the neighborhood of a trial set of parameters, so as to find corrections to the trial parameters for the next iteration (Marquardt 1959, 1963, 1966).

In the present work we take the functional relationship of interest to be

$$
\log \left(N / N_{0}\right)=f\left(z, \beta, D ; m, E_{0}, \kappa, \sigma_{0}\right)
$$

and seek a minimum value in

$$
\Phi=\sum_{i=1}^{n}\left\{\log \left(N / N_{0}\right)_{\exp }-\log \left(N / N_{0}\right)_{\mathrm{th}}\right\}^{2}
$$

All data points are assigned equal weights.

When supplied with the functional relations of the model, a set of experimental data, and a set of trial values of the parameters, the computer program yields a set of optimal parameters and an estimate of $95 \%$ confidence limits.

\section{Results}

The optimal parameters obtained by computer least squares procedures are shown in Table 2, for the cell lines listed in Table 1, with the exception of Artemia eggs (Easter and Hutchinson 1961) and budding yeast (Raju, Gnanapurani, Stackler, Madhvanath, Howard, Lyman, Manney, and Tobias 1972) for which the computer program did not converge. Since the parameters for mouse bone marrow (Broerse, Engels, LeLieveld, van Putten, Duncan, Greene, Massey, Gilbert, Hendry, and Howard 1971) and anoxically irradiated HeLa cells (Nias, Greene, Fox and Thomas 1967) were determined from X-ray and $14 \mathrm{MeV}$ neutron data, these cases were not considered in this study.

In each case several trials were made for each set of data, using as initial parameters the visually fitted set, or sets departing from the visual parameters by 10 or $20 \%$, in various combinations.

The parameters in roman type converged to identical values whatever the starting set of parameters.

The parameters in italic converged to somewhat different values, depending on the initial set of parameters. The values listed in the table are derived using visually fitted parameters as the starting point. In most cases, the other optimal parameters were within $40 \%$ of those in Table 2 .

In most cases that consistently converged to the same set of parameters, the deviation from the visually fitted values is remarkably small.

In Table 2 we list the best values of the computer-fitted parameters, the $95 \%$ confidence interval expressed as a percentage deviation, the percentage deviation from the visually fitted parameters, the number of experimental points, and the value of the product $E_{0} \sigma_{0} /$ $\mathrm{K}$, and its (RMS) uncertainty. For illustrative purposes, in Figure 1 the data for anoxically irradiated bacterial spores (Powers, Lyman, and Tobias 1968) are compared to the theoretical curves calculated from the optimal least squares parameters (heavy lines) and from the individual $95 \%$ confidence limits on each parameter. The thin lines are computed by fixing three parameters to their optimal values (e.g., $\mathrm{K}^{o}, \sigma_{0}^{o}$, and $E_{0}^{o}$ ) and varying the fourth parameter to the two values determined by the confidence interval $\left(e . g ., m^{o-}\right.$ or $\left.m^{o+}\right)$. For the eight resulting cases (denoted as $a, a^{\prime}, b, b^{\prime}$, etc.), the value of the varied parameter 


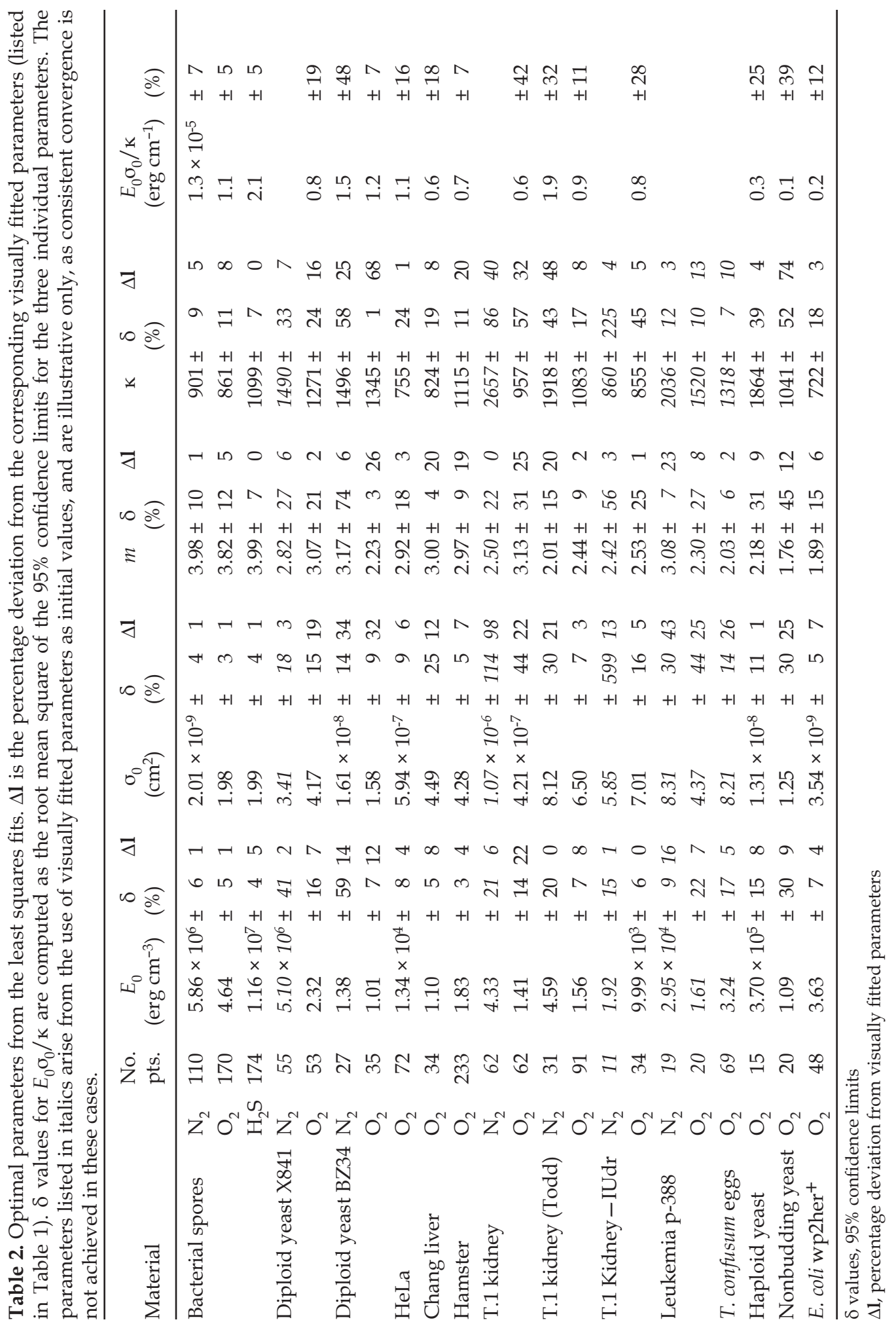


is listed at the top of the graph. For clarity, only the outermost curve is shown for each bombardment.

Where the data permit, attempts are made to determine optimal parameters from subsets of the data.

In Table 3 we explore the result of excluding data in different survival decades. Thus we exclude all data in the first decade of survival, or all data in the first and fourth (and lower) decades of survival, or retain only data in the second or in the third decade of survival. A principal result is that data in the first survival decade do not significantly affect the evaluation of parameters. This might have been anticipated, in that survival curves display a small initial shoulder. The contributions to $\Phi$ in the shoulder region are rather insensitive to variation in the parameters. In many cases the exclusion of experimental points in the first survival decade eliminates half the data, with little effect on the values of the parameters or the values of the confidence limits. The most valuable data points seem to be in the second and third decades of survival.

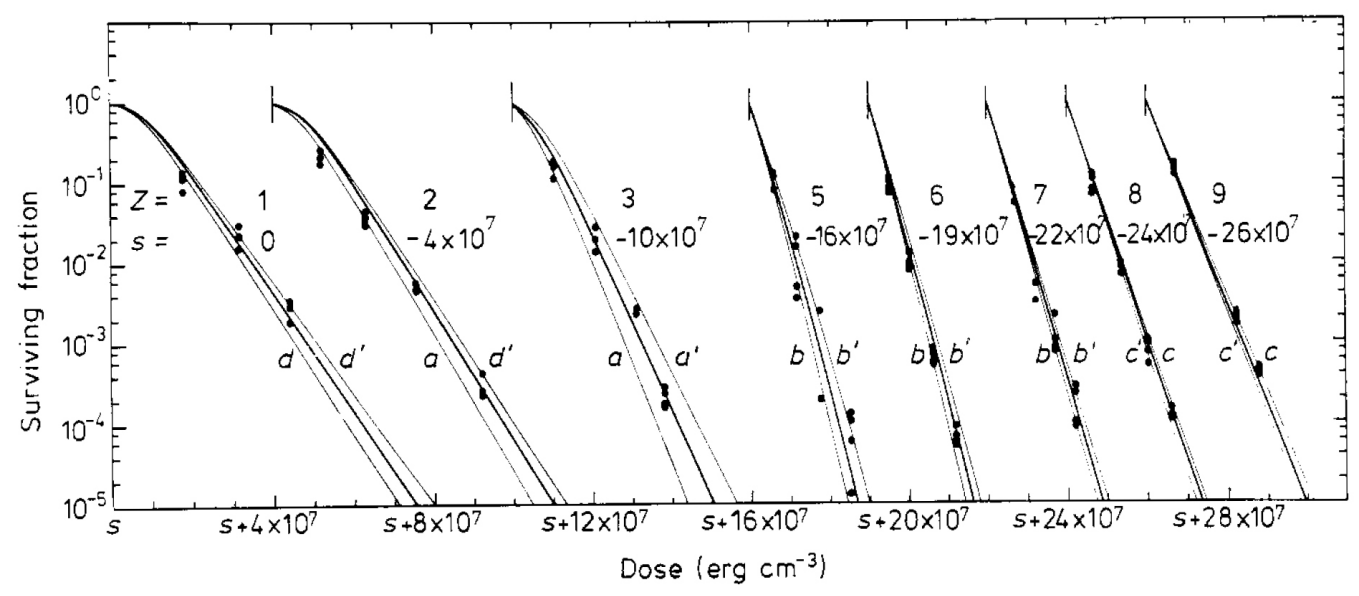

Figure 1. Calculated survivals of bacterial spores anoxically irradiated with heavy ions are compared to experimental data (Powers, private communication). The heavy lines are calculated from the optimal parameters (denoted as $m^{o}, \kappa^{o}, \sigma_{0}^{o}$, and $E_{0}^{o}$ ) and having values listed below, from the computer least squares fit. The thin lines are calculated by fixing three parameters at their optimal values $\left(e . g ., \mathrm{K}^{o}, \sigma_{0}^{o}\right.$, and $\left.E_{0}^{o}\right)$ and assigning the fourth parameter according to the $95 \%$ confidence limits $\left(e . g ., m^{o-}\right.$ or $\left.m^{o+}\right)$. These eight sets of parameters are denoted as case $a, a^{\prime}, b, b^{\prime}$, etc., and the corresponding value of the varied parameter is listed below for each case. Only the outermost curves are shown for clarity.
$m^{0}=3.98$
$\sigma_{0}^{o}=2.01 \times 10^{-9}\left(\mathrm{~cm}^{2}\right)$
$\mathrm{K}^{0}=901$
a: $\quad m^{0-}=3.57$
$E_{0}^{o}=5.86 \times 10^{6}\left(\mathrm{erg} \mathrm{cm}^{-3}\right)$
$a^{\prime}: m^{o+}=4.39$
$b: \quad \mathrm{K}^{0-}=819$
$c: \quad \sigma_{0}^{o-}=1.93 \times 10^{-9}$
$b^{\prime}: \quad \mathrm{K}^{0+}=983$
$c^{\prime}: \sigma_{0}^{0^{+}}=2.09 \times 10^{-9}$
$d: \quad E_{0}^{o^{-}}=5.52 \times 10^{6}$
$d^{\prime}: E_{0}^{o+}=6.19 \times 10^{6}$ 


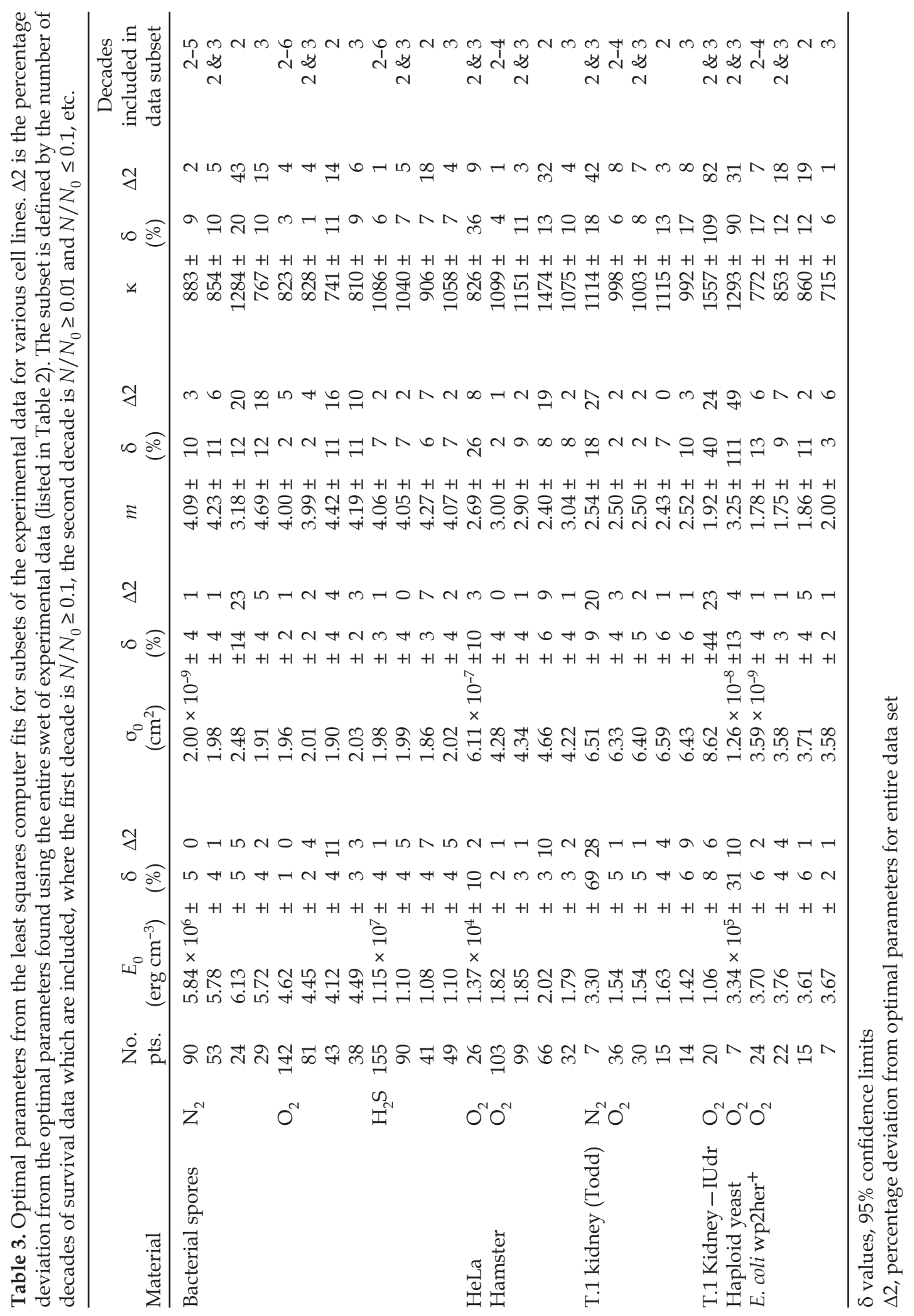




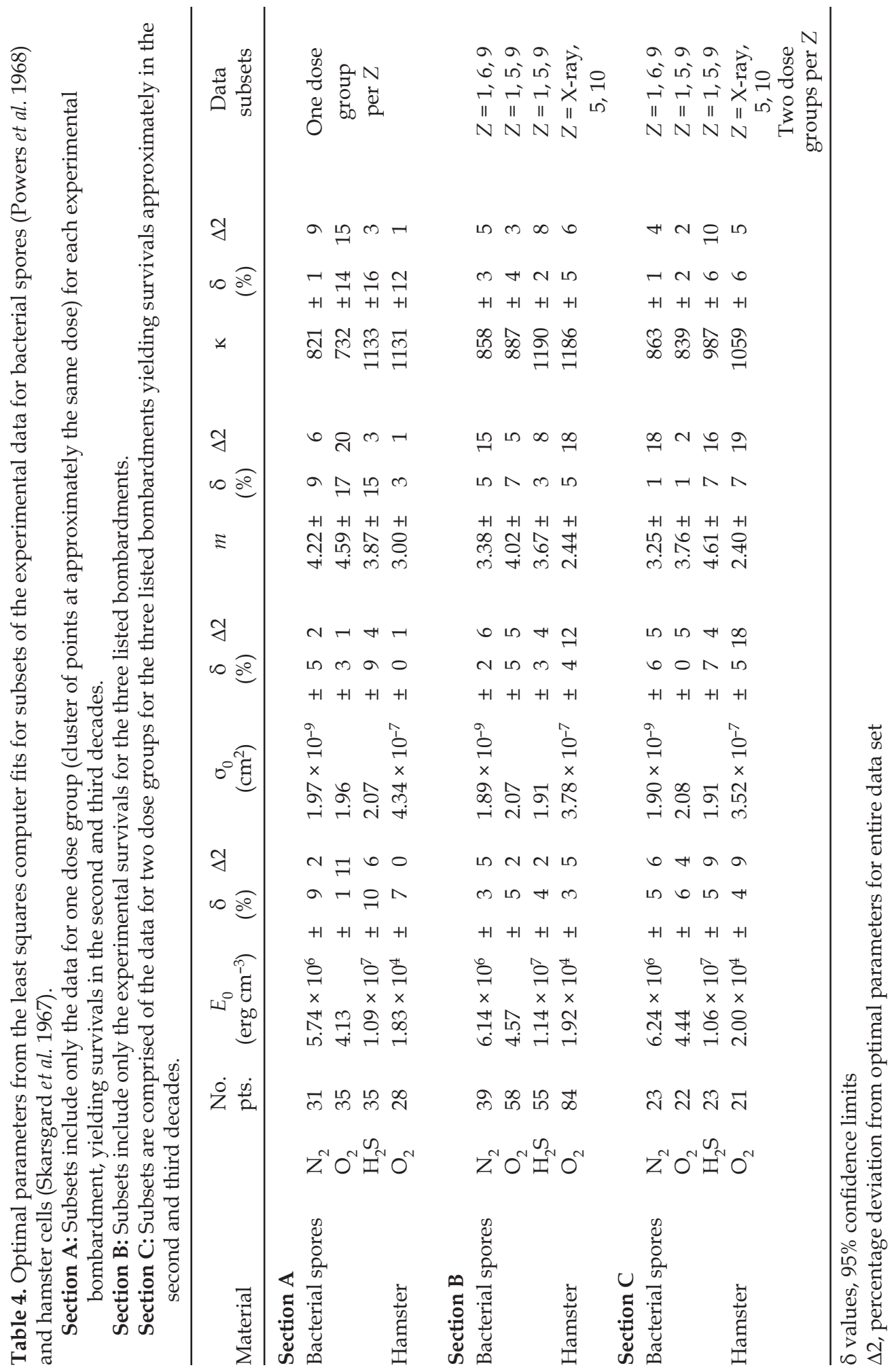


Table 4 displays the optimal parameter sets obtained by excluding data by bombardment and/or dose groupings. For these exclusions, clusters of experimental points at approximately the same dose comprise one dose group. In section A, all data are excluded except one dose group per bombardment, yielding survivals in the second to third decades. With this elimination of about three quarters of the original data, the computer finds parameters within $20 \%$ of those found using all the data.

Yet another grouping of excluded data, as suggested by the visual fitting procedures, is shown in section B of Table 4. For the case of bacterial spores (Powers et al. 1968) we have retained only the data for $Z=1,5$ or 6 , and 9, while for Chinese hamster cells (Skarsgard, Kihlman, Parker, Pujara, and Richardson 1967) we have retained the X-ray data, and data for $Z=6$ and 10. The results are similar to those in section A.

Finally we combine these exclusions. We use only two dose groups (yielding survivals in the second and third decades) per bombardment, and only three bombardments, as above, and - with about an $85 \%$ reduction in the number of experimental points - emerge with parameters again within $20 \%$ of the parameters found from all of the data, as shown in Table 4, section C.

\section{Conclusions}

This statistical study has demonstrated a remarkable consistency of theory with experiment. The procedures examined here will serve a dual function, as an alternate method of parameter evaluation, and as a tool for the design of efficient and internally consistent experiments. Our procedures require that we make a visual fit of the data as input to the computer program. Although the visual fit is somewhat subjective, we would not like to place complete reliance on the computer program. The combination of visual and computer fits to the data avoids the problems associated with sole reliance on either method, and has the advantage of a check system on parameter determination. In most cases visual parameters are very consistent with computer fits. An exception lies in the case of Chinese hamster cells, where a re-evaluation of $m$ from 2.5 to 3 may be appropriate, with an associated alteration in $\mathrm{K}$. The results of the exclusion of different decades of data in this case imply that the visual fits leaned heavily on agreement in the second decade of survival. In Figure 2 the experimental data for hamster cells (Skarsgard et al. 1967) are shown compared to the theoretical lines for calculations from the visually fitted and computer-determined parameter sets. A similar case is Chang liver cells (Todd 1975), shown in Figure 3. In each case the least squares parameters yield the better overall fit, although the differences are small.

In the data of Todd (1967) for T-1 human kidney cells anoxically irradiated, the optimal parameters are also inconsistent with the visually fitted parameters. However, the exclusion of first decade data indicates that the visual fit is probably a better choice.

The observed effect on parameter evaluation from the exclusion of data by bombardment and by decades of survival suggests the development of a protocol for efficient experimentation to minimize beam time. A survival curve after X-irradiation must be made, to yield an initial estimate of $m$ and $E_{0}$. Measurement of the diameter of the cell nucleus should yield an initial estimate of $\sigma_{0}$. An initial value of $\mathrm{K}$ may be estimated from the empirical relationship for $E_{0} \sigma_{0} / \mathrm{k}$, Tables 1 and 2. These approximations may be used to estimate the dose required to obtain surviving fractions in the second or third decade of 


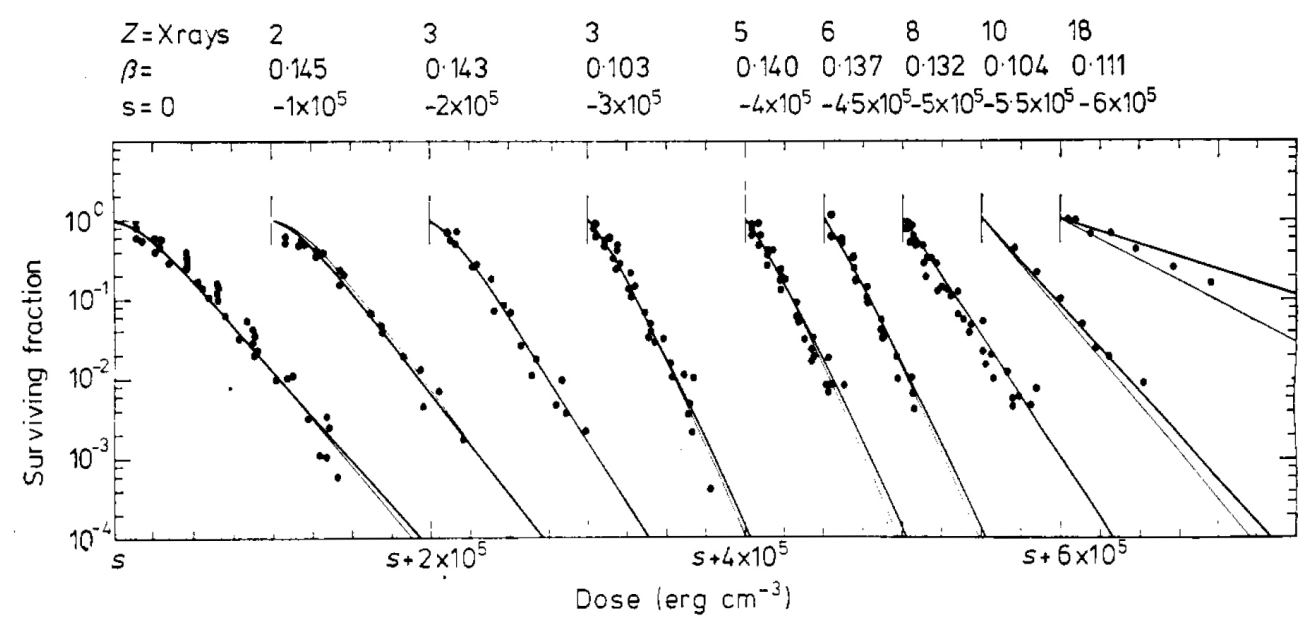

Figure 2. Calculated survivals of Chinese hamster cells irradiated with X-rays and heavy ions are compared to experimental survival data (Skarsgard, private communication). Survivals calculated from the visually fitted parameters are shown as heavy lines. The thin lines are calculated from the computer least squares parameters. Values are :

$\begin{array}{lll} & \text { heavy line } & \text { thin line } \\ m & =2.5 & 2.97 \\ \mathrm{~K} & =1400 & 1115 \\ \mathrm{\sigma}_{0}\left(\mathrm{~cm}^{2}\right) & =4.55 \times 10^{-7} & 4.28 \times 10^{-7} \\ E_{0}\left(\mathrm{erg} \mathrm{cm}^{-3}\right)= & 1.95 \times 10^{4} & 1.83 \times 10^{4}\end{array}$

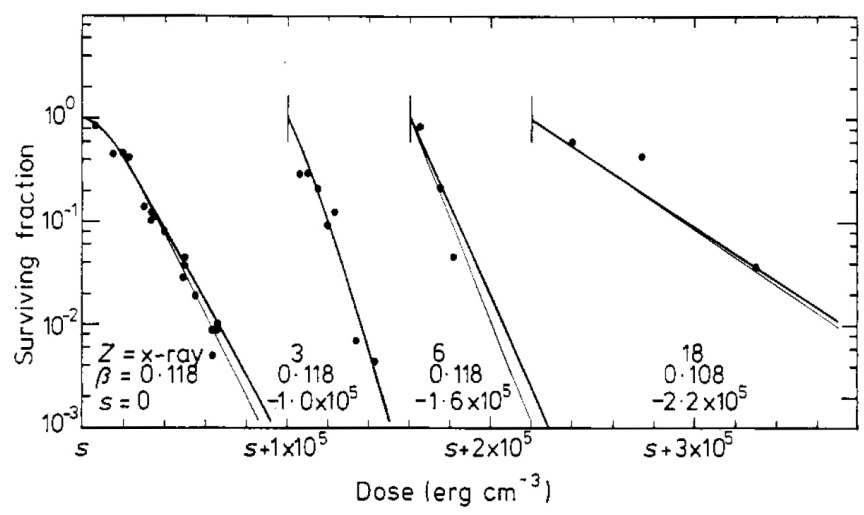

Figure 3. Calculated survivals of Chang liver cells irradiated with $X$-rays and heavy ions are compared to the experimental data of Todd (1975). Heavy and thin lines are computed as in Figure 2. Values are:

$\begin{array}{lll} & \text { heavy line } & \text { thin line } \\ m & =2.5 & 3.0 \\ \mathrm{~K} & =900 & 830 \\ \sigma_{0}\left(\mathrm{~cm}^{2}\right) & =4.0 \times 10^{-7} & 4.5 \times 10^{-7} \\ E_{0}\left(\mathrm{erg} \mathrm{cm}^{-3}\right)= & 1.2 \times 10^{4} & 1.1 \times 10^{4}\end{array}$


bombardments at $\mathrm{RBE}$ maximum $(P \simeq 0.5)$ and near the plateau value of the inactivation cross-section $(P \simeq 0.95)$. The resulting data may be used interactively with the minimization program to determine the iterations needed to determine radiosensitivity parameters to the desired precision, and to expose questionable results.

It is perhaps worthy of note that the sets of data having the largest number of experimental points (bacterial spores and Chinese hamster cells) offer the narrowest confidence limits on the parameters. In both these cases the extrapolation numbers seem remarkably close to integers, resurrecting a question long since laid to rest in target theory - Is the extrapolation number the number of cellular targets which must be hit in order to inactivate the cell?

We thank Bruce Fullerton and Christine Landkamer for their help in the course of these investigations. We also thank J. P. Ochsner and J. M. Eakman for their computer program.

This work was supported by US ERDA and the NSF (RANN).

\section{References}

Barendsen G W, Koot C J, van Kersen G R, Bewley D K, Field S B and Parnell C J 1966 Int. J. Radiat. Biol. 10317

Berry R J 1970 Radiat. Res. 44237

Broerse J J, Engels A C, Lelieveld P, van Putten L M, Duncan W, Greene D, Massey J B, Gilbert C W, Hendry J H and Howard A 1971 Int. J. Radiat. Biol. 19101

Deering R A and Rice R Jr 1962 Radiat. Res. 17774

Easter S S Jr and Hutchinson F 1961 Radiat. Res. 15333

Katz R, Ackerson B, Homayoonfar M and Sharma S C 1971 Radiat. Res. 47402

Katz R and Kobetich E J 1969 Phys. Rev. 186344

Katz R and Sharma S C 1973 Nucl. Instrum. Meth. 11193

Marquardt D W 1959 Chem. Eng. Progr. 55 (6) 65

Marquardt D W 1963 J. Soc. Indust. Appl. Math. 11 (2) 431

Marquardt D W 1966 IBM Share Library No 309401 Mortimer R, Brustad T and Cormack D V 1965 Radiat. Res. 26465

Munson R J, Neary G J, Bridges B A and Preston R J 1967 Int. J. Radiat. Biol. 13205

Nias A H W, Greene D, Fox M and Thomas R L 1967 Int. J. Radiat. Biol. 13449

Powers E L, Lyman J T and Tobias C A 1968 Int. J. Radiat. Biol. 14313

Raju M R, Gnanapurani M, Stackler B, Madhvanath U, Howard J, Lyman J T, Manney T R and Tobias C A 1972 Radiat. Res. 51310

Raju M R, Gnanapurani M, Stackler B, Martins B T, Madhvanath U, Howard J, Lyman J T and Mortimer R K 1971 Radiat. Res. 47635

Sayeg J A, Birge A C, Beam C A and Tobias C A 1959 Radiat. Res. 10449

Skarsgard L D, Kihlman B A, Parker I, Pujara C M and Richardson S 1967 Radiat. Res. Suppl. 7208

Todd P 1964 Ph.D. Thesis University of California, Berkeley, California 94720 Todd P 1967 Radiat. Res. Suppl. 7196 
Todd P 1975 Radiat. Res. 61288

Yang C, Craise L, Welch G P and Tobias C A 1973 Radiat. Res. 55523

Yang C, Craise L, Welch G P and Tobias C A Donner Laboratory Report Lawrence Berkeley Laboratory LBL-2016 Berkeley, CA 94720 